0.74-1.12, $\mathrm{p}=0.37$ ), respectively. In contrast, "ever-users" of sBB had a significantly elevated risk for recurrence and death in multivariate analysis (PFS HR 1.22 95\%-CI 1.05-1.41, $\mathrm{p}=0.009$; OS HR 1.25 95\%-CI 1.06-1.47, $\mathrm{p}=0.009$ ).

Conclusion* In this large pooled analysis neither a co-medication with metformin nor with statins had a significant impact on survival in patients with primary ovarian cancer. In contrast, co-medication with a beta-blocker was associated with worse survival. Further studies are warranted to confirm this observation.

\section{PARP-INHIBITORS BEYOND PROGRESSION: A NEW WAY TO MANAGE OLIGOMETASTATIC OVARIAN CANCER RECURRENCE}

${ }^{1} \mathrm{C}$ Marchetti ${ }^{*},{ }^{1} \mathrm{E}$ Palluzzi, ${ }^{1} \mathrm{~S}$ Cappuccio, ${ }^{1} \mathrm{G}$ Avesani, ${ }^{1} \mathrm{~A}$ Nardangeli, ${ }^{1,2} \mathrm{G}$ Scambia, ${ }^{1,2}$ A Fagotti. ${ }^{1}$ Fondazione Policlinico Agostino Gemelli-IRCCS, Dipartimento di Scienze della Salute della Donna, del Bambino e di Sanità Pubblica, Rome, Italy; ${ }^{2}$ Università Cattolica del Sacro Cuore, Rome, Italy

\subsection{6/ijgc-2021-ESG0.367}

Introduction/Background* The benefit of surgery and maintenance treatment with parp inhibitors (PARPi) has been recently shown in ovarian cancer (OC) recurrence. Also, the efficacy and safety of stereotactic body radiotherapy (SBRT) is demonstrated in patients with metastatic, persistent, and recurrent OC. The management of oligometastatic progression (OMP) during PARPi maintenance is unclear and continuing the treatment beyond progression could be an option.

Methodology This is an observational, retrospective, single arm study. Patients affected by OC recurrence treated with PARPi

\begin{tabular}{ll} 
Abstract 242 Table 1 & \\
\hline & PARPi manteinance \\
\hline & Nr. (\%) \\
All patients & 24 \\
Median age (years) (range) & $51(35-67)$ \\
Histology & \\
Serous & $24(100)$ \\
PARPi & \\
Olaparib & $9(38)$ \\
Niraparib & $15(62)$ \\
BRCA status & \\
BRCA MT & $10(42)$ \\
BRCA WT & $14(58)$ \\
Therapy at oligometastatic progression & \\
Surgery & $9(38)$ \\
SBRT & $15(62)$ \\
Site of oligometastatic progression & \\
Lynphnode & $14(58)$ \\
Peritoneal & $4(17)$ \\
Parenchymal disease & $6(25)$ \\
PFS & Months \\
Median PFS1 & $(\mathrm{Cl})$ \\
& 23 months \\
Median ppPFS & $(\mathrm{Cl} 95 \% 11-34)$ \\
Median bpPFS & 6 months \\
& $(\mathrm{Cl} 95 \% 5-7)$ \\
\hline & 29 months \\
(CI 95\% 17 - 40) \\
\hline
\end{tabular}
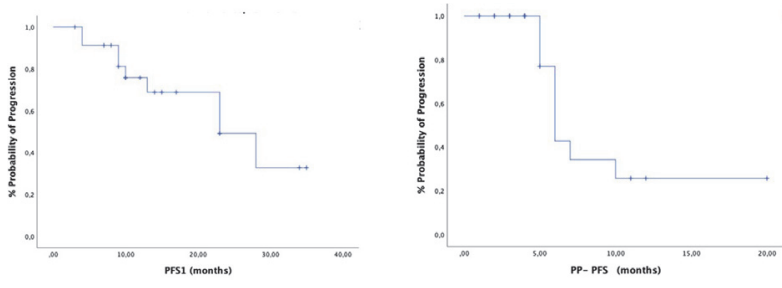

Abstract 242 Figure 1

in maintenance setting received surgery or SBRT, if OMP occurred. OMP was assessed by either CT scan or PET/CT scan, in case of isolated disease progression (one nodule) or discrete diffusion (up to three nodules in different locations) or progression in "sanctuary" site. Maintenance treatment was continued until extensive progression of disease. Primary objectives were: Progression Free Survival 1 (PFS1), defined as the time elapsed from the start of PARPi and OMP; post-progression-PFS (ppPFS), defined as the time elapsed from OMP and the last follow up (FU). Beyond-progression PFS (bpPFS), defined as the time elapsed from the start of PARPi and the definitive progression of disease or last FU (PFS1+ppPFS), and efficacy of surgery versus SBRT at OMP were secondary objectives.

Result(s)* From June 2017 to December 2020186 OC patients were treated with PARPi maintenance at recurrence. Of these $24(13 \%)$ developed OMP (58\% lymphnodes, 17\% peritoneal, $25 \%$ visceral disease). Median age was 51 years. Olaparib and Niraparib maintenance were administered to 9 (38\%) and 15 (62\%) patients, respectively. Median PFS1 was 23 months [Confidence Interval (CI) 95\% 11 - 34]. When OMP occurred $9(38 \%)$ and $15(62 \%)$ pts were subjected to surgery and SBRT, respectively. Median ppPFS was 6 months (CI 95\% 5 - 7). At the time of this publication $62.5 \%$ patients are still on treatment with PARPi beyond progression. Conclusion* OC patients, who have an OMP during PARPi maintenance at recurrence, may continue to benefit from PARPi treatment if combined with local treatment. Molecular assessment at oligometastatic and extensive progression could provide further information to define PARPi resistance mechanisms according to the type of disease progression.

\section{PFS OF ELDERLY OVARIAN CANCER PATIENTS MIGHT BE PREDICTED BY G-8 GERIATRIC SCREENING TOOL - RESULTS OF A RETROSPECTIVE COHORT STUDY}

${ }^{1} \mathrm{~K}$ Anic*, ${ }^{1} S$ Birkert, ${ }^{1} \mathrm{R}$ Schwab, 'MW Schmidt, ${ }^{1} \mathrm{~V}$ Linz, ${ }^{1} S$ Krajnak, ${ }^{1} \mathrm{~A}-\mathrm{S}$ Heimes, ${ }^{1} \mathrm{M}$ Schmidt, ${ }^{2} \mathrm{C}$ Westphalen, ${ }^{3}$ EK Hartmann, ${ }^{1} \mathrm{~A}$ Hasenburg, ${ }^{1} \mathrm{M}$ Battista. ${ }^{1}$ University Medical Centre of the Johannes Gutenberg University Mainz, Department of Gynaecology and Obstetrics, Mainz, GERMANY; ${ }^{2}$ University Medical Centre of the Johannes Gutenberg University Mainz, Department of Geriatric Medicine, Mainz, GERMANY; ${ }^{3}$ University Medical Centre of the Johannes Gutenberg University Mainz, Department of Anesthesiology, Mainz, GERMANY

\subsection{6/ijgc-2021-ESG0.368}

Introduction/Background* The aim of this study was to evaluate the impact of the preoperative global health status on the prognosis of patients with ovarian cancer (OC) older than 60 years, who received cytoreductive surgery.

Methodology G-8 geriatric screening tool (G-8 score), Lee Schonberg prognostic index, Eastern Cooperative Oncology Group (ECOG) performance status and Charlson Comorbidity Index (CCI) were determined retrospectively in a consecutive 